\title{
Improving Breakdown Voltage Characteristics of GDAs using Trigger Voltage
}

\begin{abstract}
Sei-Hyun Lee ${ }^{\dagger}$
Abstract - This paper investigates a method to improve the breakdown voltage characteristics of a gas discharge arrester (GDA) for a surge suppressor. The middle electrode is inserted between two terminal electrodes. Voltage application to the electrode synchronized and amplified by the impulse voltage decreases spark overvoltage from $45 \%$ to $57.6 \%$. The decrease is caused by higher voltage slope, as opposed to applied impulse voltage (by 5.5 to 6.2 times). In addition, the GDA model using ATPDraw was used to analyze the operation characteristics of GDAs. The test and simulation results agree to within $2 \%$ when the trigger source was used.
\end{abstract}

Keywords: Gas discharge arrester (GDA), Breakdown voltage, Trigger voltage, dv/dt, surge, ATPDraw

\section{Introduction}

The use of integrated circuits in several technological applications has resulted in the rapid development of the electronics industry. Integrated technology has provided faster transmission and processing of digital data, as well as offered additional operational features. However, modern integrated electronics are very vulnerable to power abnormalities and, in particular, to power surges that cause damage (i.e., either partial or complete disruption of the network) and service interruption [1], [5]. The surgeinduced interference from an electrical communication facility can cause significant economic damage. Protection against surges is critical, and hence, research and development of surge protectors is very important. One effective and economical approach to protect equipment from transient abnormal voltage is to limit the voltage under the insulation level of the protected equipment by installing surge suppressors at the input and output terminals. Gas discharge arrester (GDA) protectors are one of the oldest methods used against transient overvoltages in a circuit. Typical miniature spark gaps in a ceramic case can conduct transient current pulses of 5-20 kA for tens of microseconds without significant damage to the spark gap [2].

Spark gaps offer the smallest shunt capacitance of all known nonlinear transient protection devices, with typical capacitance between 0.5 and $2 \mathrm{pF}$. Low capacitance categorizes spark gaps as one of few nonlinear devices for use in circuit protection; their signal frequency is greater than 50 $\mathrm{MHz}$ [2]. GDAs with self-restoring characteristics are used in high frequency network interface devices to protect against lightning and alternating current power cross faults in telecommunication networks [3]. GDAs are robust and

\footnotetext{
$\dagger$ Corresponding Author: Dept. of Electrical Measurement and Control, Korea Polytechnic College IV. (phdsh@kopo.ac.kr) Received: May 7, 2010; Accepted: July 21, 2010
}

relatively inexpensive; moreover, they do not limit the bandwidth of high-frequency circuits as much as other nonlinear shunt components [3]. Compared with solid state protectors, GDAs can carry much higher currents. However, some major disadvantages have prohibited the use of GDAs in telecommunication applications involving sensitive equipment [1]. First, its use requires relatively high electric field for plasma ignition, which suggests a slowed down GDA operation resulting in high residual load voltages. Second, GDAs are difficult to turn off after a transient has ended, consequently causing temporary power interruption to the site. Lastly, the spark developed between electrodes in GDAs can damage the sensitive equipment. In relation, this power surge-sensitive equipment can be destroyed by higher breakdown voltages.

Currently, nonlinear surge protective devices are used to protect equipment from such damaging overvoltages. An efficient transient protection system should contain a protection circuit, which combines different surge protectors in order to gain their individual advantages [4]. Proper coordination of these protectors with other circuit components is essential to obtain adequate performance protection. If the breakdown voltage of GDAs becomes too low, the rated voltage of the nonlinear device that limits the residual voltage decreases. As a result, some of the devices connected with GDAs in series and in parallel (i.e., to limit overvoltage) can be omitted. In this paper, a trigger circuit is used to decrease the breakdown voltage of GDAs. Apart from an impulse test voltage applied to the terminal, a trigger voltage amplified and synchronized on the test voltage is placed simultaneously to the middle electrode. We measured and analyzed breakdown voltages at the electrode terminals independent of trigger voltage and created a GDA model to simulate GDA using ATP-Draw. Test results are then compared with simulation results. 


\subsection{Operating mode of the GDA}

In general, a spark-over occurs whenever the surge voltage exceeds the electrical strength of the system insulation. This discharge can limit surge voltage and reduce interference energy at a short period [6]. The electrical breakdown process of gases in uniform fields exhibits two phases: prebreakdown and breakdown ionization. When the applied voltage is greater than the spark potential, the generalized phenomenon of breakdown, as described by Townsend [7], would include (i) primary ionization; (ii) secondary ionization, electron attachment, and detachment; and (iii) ion conversion processes in collisions [7].

The behavior of GDAs (Fig. 1) can be divided into four operating domains:

Range a: There are virtually no current flows, as the voltage rises to the dynamic breakdown voltage of the GDA. This domain is characterized by an almost infinite insulation resistance ( $\mathrm{R}>1$ gigaohm). When applied voltage exceeds DC breakdown voltage, the processes preceding gas discharge between electrodes begins. Free electrons are accelerated by increasing electric-field strength (E). With sufficient kinetic energy, these electrons ionize atoms and cause secondary electron emission from the cathode [9]. Actual gas discharge occurs when the voltage reaches the value of the dynamic breakdown voltage $(\mathrm{Vd})$. The time for the voltage to change from Vs to Vd is referred to as "statistical time delay of discharge" (Td). This value can be determined from the responses of the measurements performed on the GDA. The mathematical formulation of $\mathrm{Td}$ is given as [8]

$$
T_{d}=a \cdot S^{-b}[\mathrm{~s}]
$$

where $T_{d}$ is the delay time, $S$ is the voltage steepness of the front wave expressed in volts per second, and $a$ and $b$ are empirical coefficients for each type of GDA.

Range b: After ignition, the voltage drops to the glow voltage level (90 to $300 \mathrm{~V}$, depending on type, with current of $0.2 \mathrm{~A}$ up to about $1.5 \mathrm{~A}$ ) [2]. When a conductive path between the electrodes is established, a streaming discharge occurs, causing the gas between the electrodes to become ionized. The passing current is then increased, causing the diameter of the conductive stream to rise; subsequently, secondary ionization of the gas is also increased. In the final phase, the stream changes into an electric arc. This rising current can be modeled by changing arc resistance in accordance with the Toepler equation [4], [8]:

$$
R(t)=\frac{K \cdot d}{\int_{0}^{t} i d t}
$$

where $K$ is the Toepler constant. Incidentally, $0.005 \pm 20 \%$ $\mathrm{Vs} / \mathrm{m}$ is not constant; the value changes depending on gas composition and pressure, as well as on distance $d$ between electrodes [9]. The denominator in Eq. (2) represents electric charge (electrons, ions) transmitted over a time-period through the stream. In the breakdown phase, the electric current between electrodes rises steeply, and simultaneously, resistance decreases according to Eq. (2). When the current exceeds a certain value, the stream turns into an electric arc.

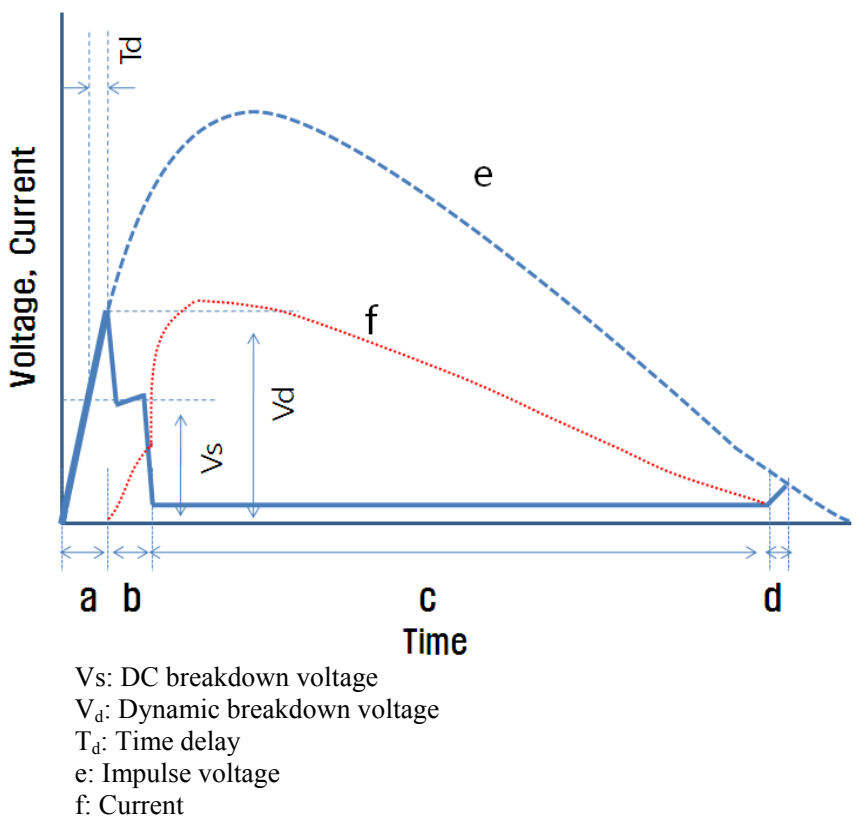

Fig. 1. Impulse voltage and discharge mode as a function of time at the GDA.

Range c: With further increase in current, transition occurs at the arc range. The extremely low arc voltage of 10 $35 \mathrm{~V}$ that is typical for this mode is virtually independent of the current over a wide range of voltages. As current increases, GDA shifts from glow voltage to arc voltage. The GDA is most effective in this domain because the discharged current can reach several thousand amperes without any commensurate increase across the terminals of the arc voltage. The conductive channel is composed of a plasma consisting of hot ionized gases; the main source of the electric charge carriers corresponds to the thermionic emission of the electrons from the hot cathode $(\mathrm{T} \approx 3000 \mathrm{~K})$ [10].

Range d: As overvoltage decreases, the current through the GDA decreases accordingly until it drops below the minimum value necessary to maintain the arc. When the current falls below $0.03 \mathrm{~A}$, thermionic emission ends, causing the cathode to cool down. Consequently, the arc discharge stops immediately; after passing through the glow range, the GDA extinguishes at a voltage above a certain range. In the applied test voltage that is set roughly equal to glow voltage $(\approx 100 \mathrm{~V})$, the GDA recovers its initial insulating properties [11]. 


\section{Experimental Setup and Procedure}

Fig. 2 shows the configuration of the GDA. Labels (A) and (C) in the experimental setup are for brass metal electrodes. Discharge was carried out in the Pyrex tube. After the tube was evacuated by a vacuum pump, nitrogen gas $(99.99 \%)$ was inserted through a moisture adsorption meter. The pressure in the tube was 0 Torr at room temperature. Label (B) represents the middle electrode where high trigger voltage was applied. The distance between electrodes (A) and (C) was $0.5 \mathrm{~mm}$.

The schematic of the impulse generator for the experiment is shown in Fig. 3. The charged voltage in capacitor $\mathrm{C} 1$ was applied to the GDA using switch S1. In addition, a trigger voltage was amplified and synchronized to high voltage capacitor $\mathrm{C} 2$ and transformer $\mathrm{Tr}$.

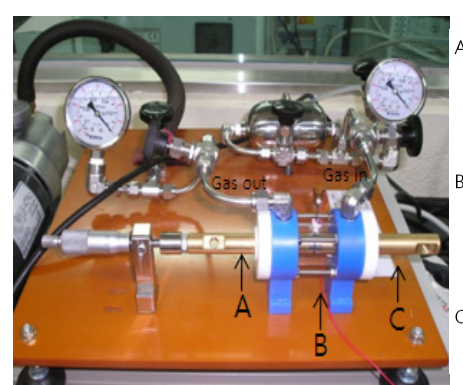

(a) Test equipment

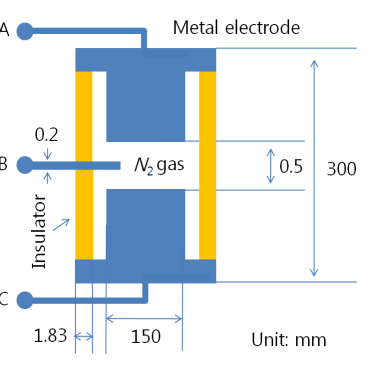

(b) Cross-section of the GDA
Fig. 2. Configuration of the GDA.

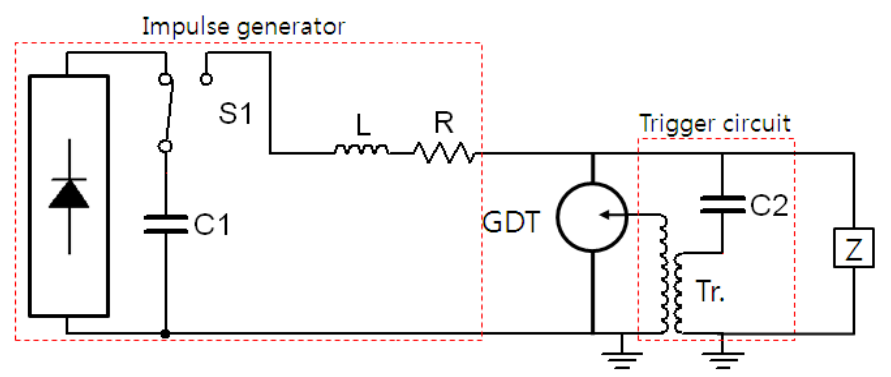

Fig. 3. Schematic of impulse generator voltage wave.

$\begin{array}{ll}\text { S1 } & \text { Making switch } \\ \mathrm{C} 1 & \text { Capacitor for charging } \\ \mathrm{L} & \text { Inductance of the line } \\ \mathrm{R} & \text { Resistance of the line } \\ \mathrm{C} 2 & \text { Shunt capacitor, } 1 \mathrm{nF}, \text { Working } 6 \mathrm{kV} \\ \mathrm{Tr} . & \text { Transformer, } \\ & \text { Primary } 10 \mu \mathrm{H}, 112 \mathrm{~m} \Omega \\ & \text { Secondary } 14.7 \mathrm{mH}, 38.7 \Omega \\ \text { GDA } & \text { Gas discharge arrester }\end{array}$

The slope of the amplified voltage was about 5.6 times greater than the applied voltage. The amplified voltage was applied to the middle electrode and discharged before the applied voltage reached the breakdown voltage.

The applied voltage corresponds to an impulse with a positive voltage waveform of $1.2 / 50 \mu$ s. The experimental procedure that involved applying an impulse voltage to the middle electrode was not connected. During this phase, both GDA terminal voltage and the secondary winding were measured.

The procedure was repeated after the middle electrode was closed. GDA peak voltage was compared with the case prior the application of trigger voltage.

\section{Results and Discussion}

Fig. 4 shows the applied impulse voltage wave with a slope of $18.5 \mathrm{kV} / \mu \mathrm{s}$ and a peak voltage of $4 \mathrm{kV}$. Experimental equipment was discharged between 100 and $300 \mathrm{~ns}$. When impulse voltage was applied to the GDA, the secondary voltage of the transformer decreased with decaying oscillation. To visualize the slope of the voltage wave amplified by the capacitor and the transformer, the voltages were extended up to $200 \mathrm{~ns}$. For the extended wave after $50 \mathrm{~ns}$, an increase in difference between trigger voltage and applied impulse voltage, as exhibited by the voltage slope, could be observed. The first peak voltage was used as the trigger. The applied impulse voltage and the voltage of the amplified slope are shown in Fig. 5. In Fig. 5(A), notations (a) through (d) are the synchronized and amplified voltage profiles corresponding to impulse voltages (e)-(h) in Fig. 5(B); these were subsequently applied in the GDA. Table 1 presents the raw data. Note that the slope of the amplified voltage increased by 5.5 to 6.2 times.

Fig. 6 compares the results when the trigger voltage is applied and not applied to the middle electrode. Fig. 6(a) is the trigger voltage resulting from the applied impulse voltage. The trigger voltage is the secondary voltage of the transformer when the middle electrode is not connected to the trigger electrode. Fig. 6(b) is the terminal voltage of the GDA when the trigger voltage is not applied. Fig. 6(c) is the trigger voltage when the middle electrode is connected to the trigger electrode. Finally, Fig. 6(d) is the terminal

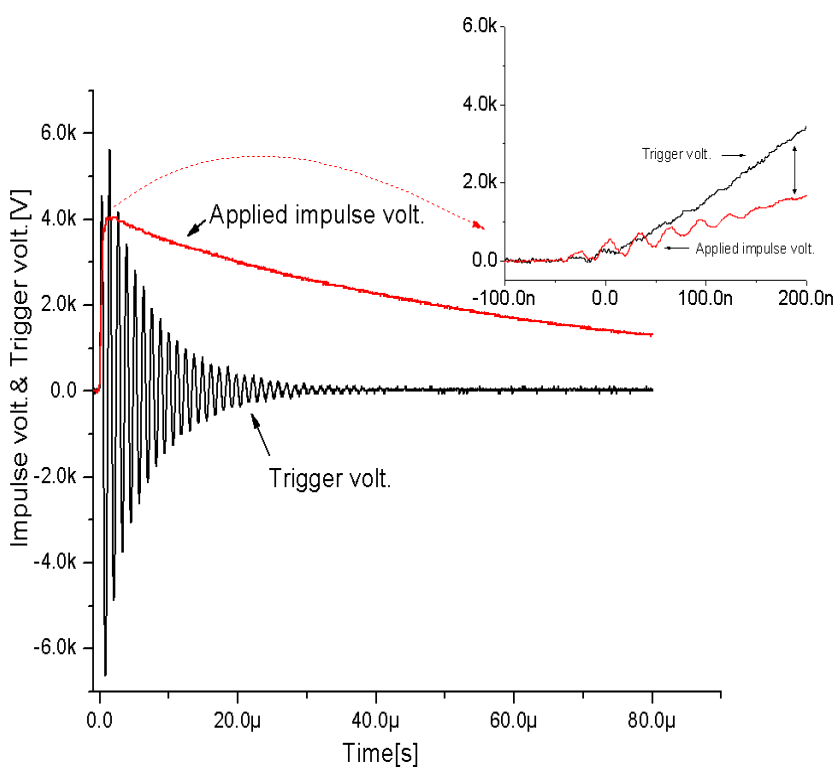

Fig. 4. Test voltage applied to the GDA. 


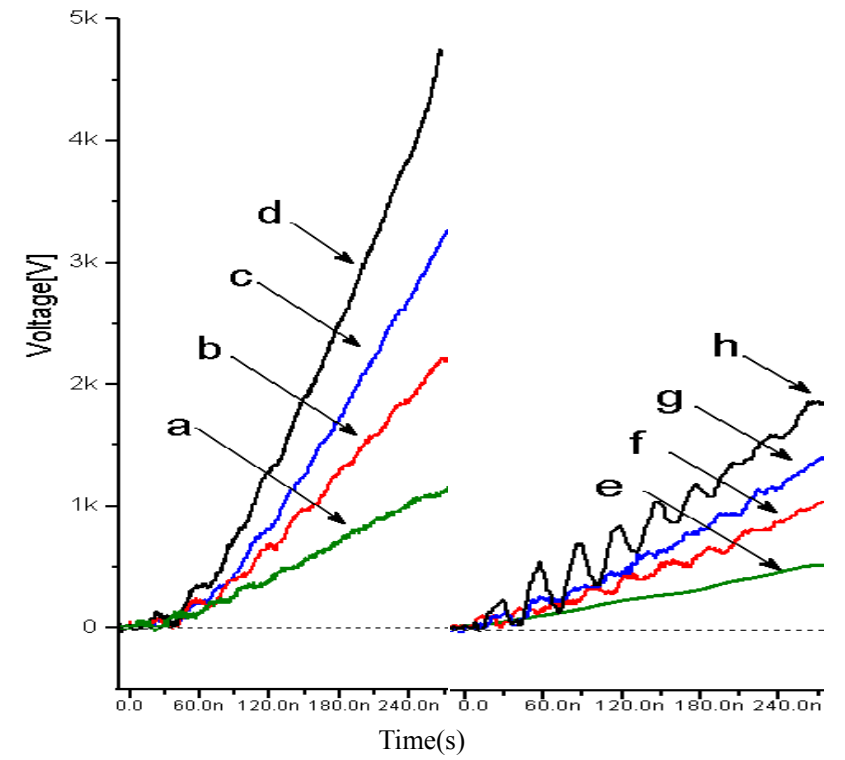

(A) After amplification

(B) Before amplification

Fig. 5. The slope of the voltage.

Table 1. Voltage slopes

\begin{tabular}{c|c|c|c|c}
\hline Trigger volt. & (a) & (b) & (c) & (d) \\
\hline $\mathrm{kV} / \mu \mathrm{s}$ & 18.5 & 15.6 & 9.8 & 4.4 \\
\hline Test volt. & (e) & (f) & $(\mathrm{g})$ & $(\mathrm{h})$ \\
\hline $\mathrm{kV} / \mu \mathrm{s}$ & 3.3 & 2.5 & 1.7 & 0.8 \\
\hline$\Delta$ volt. & 5.6 times & 6.2 times & 5.7 times & 5.5 times \\
\hline
\end{tabular}

voltage of the GDA when trigger voltage is applied. This terminal voltage is a very important parameter because it can affect the protection characteristics of devices.

In Fig. 6(b), the breakdown voltage of the GDA is 1825 $\mathrm{V}$ at 511 ns. However, in Fig. 6(d), the breakdown voltage of the GDA is $1137.5 \mathrm{~V}$ at $275 \mathrm{~ns}$, corresponding to a reduction in breakdown voltage and breakdown time of $687.5 \mathrm{~V}$ and $236 \mathrm{~ns}$, respectively. Therefore, the effects of applying the trigger voltage to the middle electrode are evident. In Fig. 6(c), the trigger is activated at $918 \mathrm{~V}$ at 163 ns. The breakdown voltage of the GDA, compared with the activated trigger voltage, increased by as much as $219.5 \mathrm{~V}$. Response time lagged by as much as 112 ns. Several reasons have been provided to explain the results. First, breakdown was initiated between the middle electrode and the other electrodes, but sufficient ionization was not generated. Second, even when the ionization between the middle electrode and the other electrodes was sufficient, the electric field discharge between the end electrodes of the GDA was weak. Third, although the electric field between the end electrodes of the GDA and the power of the trigger source to induce ionization were sufficient, it required a time delay to stimulate avalanche and arcing. This mechanism is called "statistical time delay of discharge." However, if the breakdown of the GDA occurred simultaneously with the initiation of trigger discharge, the breakdown voltage of the GDA would decrease as much as 907 $\mathrm{V}$, and the time to breakdown would decrease as much as
348 ns. Fig. 7 shows the results of breakdown voltage and voltage slope, both with and without an applied trigger voltage. The dotted line corresponds to the regression curve achieved by curve-fitting.

The left axis shows the terminal voltage of GDA. Curve (b) is the voltage of the middle electrode and curve (c) is the voltage after the impulse voltage is applied, given that the trigger voltage has also been applied.
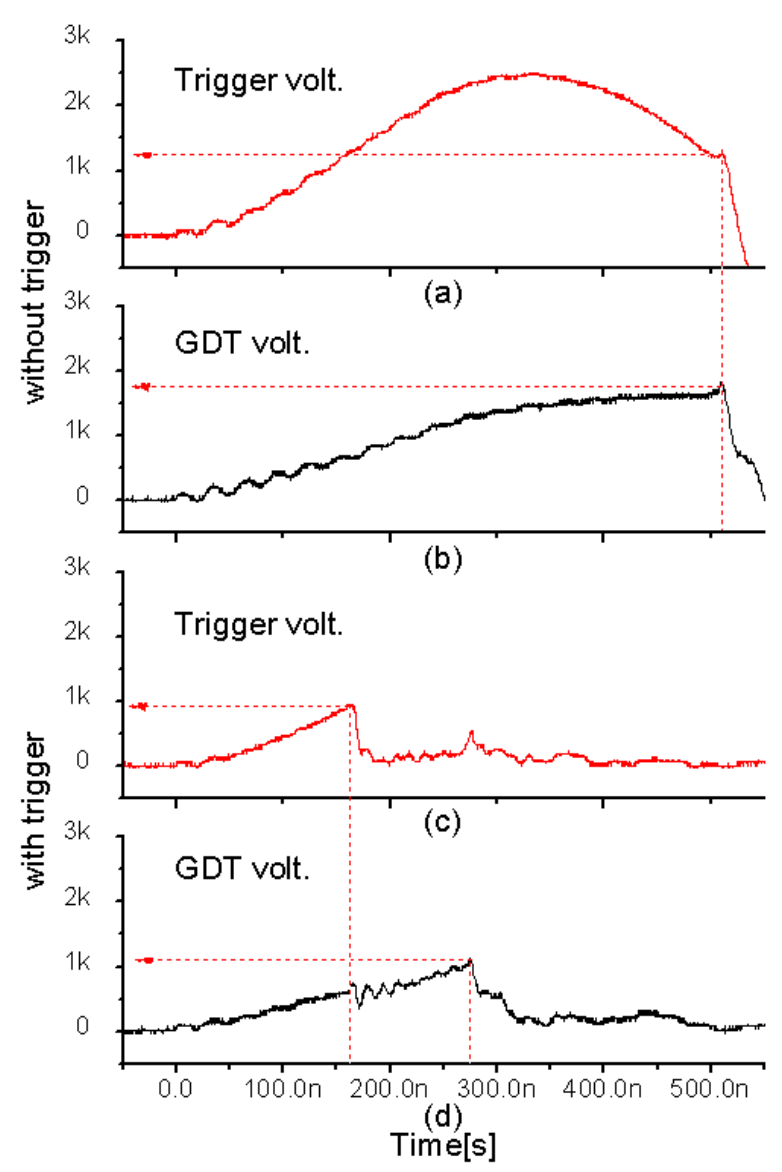

Fig. 6. Example of the measured results.

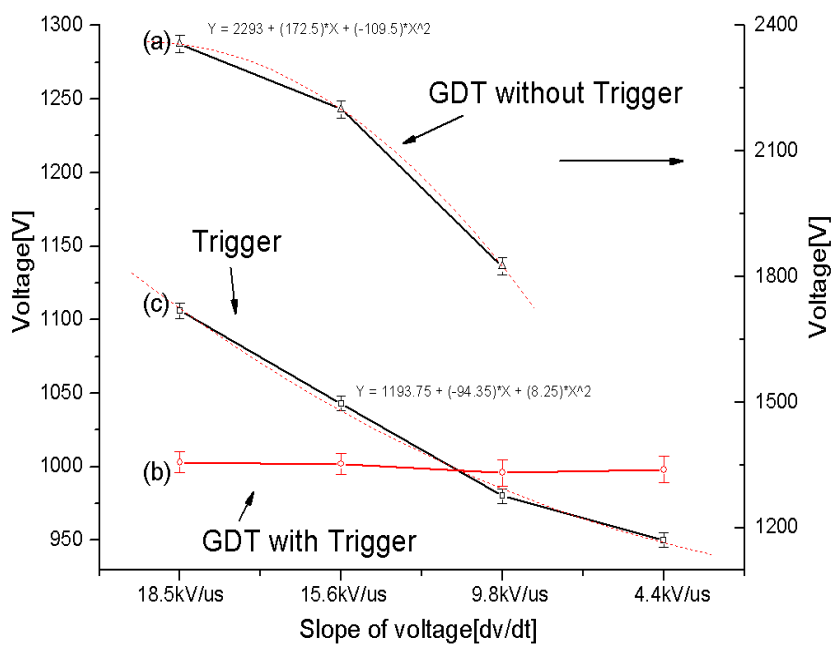

Fig. 7. Breakdown voltage vs. slope of trigger voltage. 
The right axis is the terminal voltage of the GDA. Curve (a) is the voltage after the impulse voltage is applied without application of the trigger voltage. The $\mathrm{x}$-axis is the slope of the trigger voltage. In curve (a), at voltage slope $(\mathrm{dv} / \mathrm{dt})=18.5 \mathrm{kV} / \mu \mathrm{s}$, breakdown voltage is $2356 \mathrm{~V}$. As voltage slope decreases, the breakdown voltage would decrease as well. At $\mathrm{dv} / \mathrm{dt}=9.8 \mathrm{kV} / \mu \mathrm{s}$, breakdown voltage discharge is at $1825 \mathrm{~V}$. However, discharge is not generated at slope $=4.4 \mathrm{kV} / \mu$ s with peak voltage $=1 \mathrm{kV}$ because the electric strength for the insulation breakdown at the gap between the electrodes is too weak.

In this experiment, the minimum breakdown voltage was $1825 \mathrm{~V}$ for a fixed electrode distance and a given $\mathrm{dv} / \mathrm{dt}$, gas pressure, and gas type. The voltages obtained when the trigger voltage was applied to the middle electrode (see left axis, Fig. 6). In curve (c), at $\mathrm{dv} / \mathrm{dt}=18.5 \mathrm{kV} / \mu \mathrm{s}$, the breakdown voltage between the middle electrode and others is $1106 \mathrm{~V}$. As voltage slope decreases, the breakdown voltage decreases linearly. However, in curve (b), because a trigger voltage is applied, the breakdown voltages of the GDA are generated at around $1000 \mathrm{~V}$ independent of voltage slope. Thus, breakdown voltage induced when using the trigger can be lowered by up to $1356 \mathrm{~V}$ compared with the results in curve (a).

Discharge voltage must also be decreased because of the larger $\mathrm{dv} / \mathrm{dt}$ of the trigger voltage used between the middle electrode and the other electrodes compared with the voltage applied at the terminal of GDA. However, in curve (c), when voltage slope $=4.4 \mathrm{kV} / \mu \mathrm{s}$, the trigger discharge is initiated at $950 \mathrm{~V}$, but the terminal voltage of the GDA is even higher at $1003 \mathrm{~V}$. When voltage slope $=9.8 \mathrm{kV} / \mu \mathrm{s}$, the trigger discharge is initiated at $980 \mathrm{~V}$, but the terminal voltage of the GDA is also higher at $1000 \mathrm{~V}$.

This phenomenon occurs because of the required time to initiate an electron avalanche [2], [11]. In this case, the discharge by the applied trigger voltage is initiated. However, if the electric strength between the terminal electrodes of the GDA after triggering is not strong enough, the actual breakdown voltage becomes higher than trigger voltage. Therefore, triggering is rendered ineffective under this condition.

Fig. 8 shows the results of response time and voltage slope. Curve (b) corresponds to the applied trigger voltage is applied whereas curve (a) is for the unutilized trigger voltage. Curve (c) refers to initiation times when discharge is generated between the middle electrode and the negative electrode after applying the impulse voltage. Trigger voltage has been applied in curve (c). The regression curves generated by curve-fitting are shown as dotted lines. Response time decreases asymptotically with increase in voltage slope. However, a decrease in voltage slope results in an exponential increase in time.

Clearly, by comparing curves (a) and (b) in Fig. 8, the initiation time of the breakdown at 9.8, 15.6, and 18.5 $\mathrm{kV} / \mu \mathrm{s}$ is decreased by 234,144 , and $79 \mathrm{~ns}$, respectively, when trigger voltage is applied to the middle electrode. By comparing curves (b) and (c), the initiation times for the breakdowns at $4.4,9.8,15.6$, and $18.5 \mathrm{kV} / \mu \mathrm{s}$ is decreased

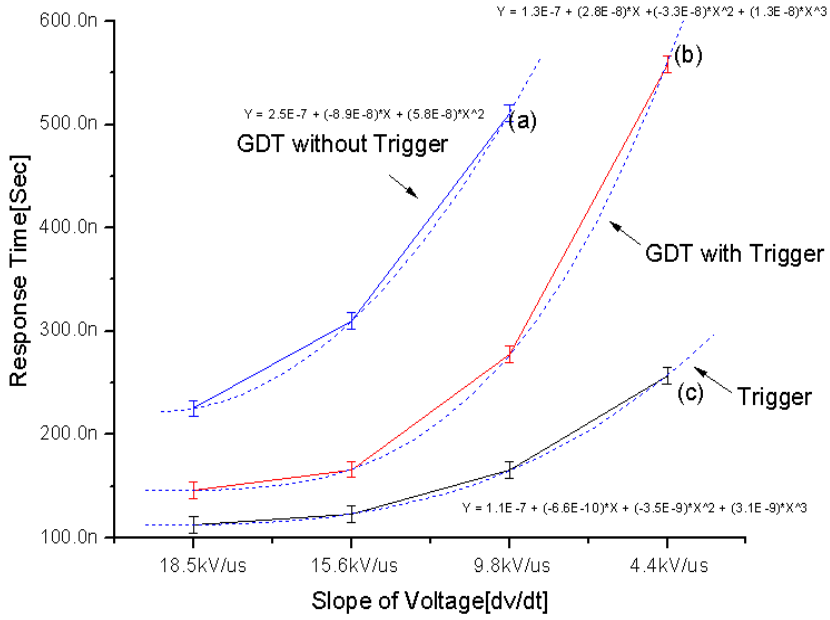

Fig. 8. Response time vs. slope of the trigger voltage.

by $301,112,43$, and $34 \mathrm{~ns}$, respectively, when the trigger voltage is applied to the middle electrode. Thus, time differences decrease significantly as voltage slope increases. Based on the regression curves shown in Figs. 7 and 8 (i.e., the first order equation to predict response time $T_{R}$ ), voltage slope and dynamic breakdown voltage $\left(\mathrm{V}_{\mathrm{d}}\right)$ can then be obtained from Eqs. (3) and (4).

$$
\begin{gathered}
T_{R}=8.242 E-6-3.205 E-17 \cdot(d v / d t)+\left(-7.568 E-9 \cdot V_{d}\right) \\
V_{d}=1007.016-5.141 E-10 \cdot(d v / d t)+\left(-2819030.142 \cdot T_{R}\right)
\end{gathered}
$$

\section{Simulation}

In this paper, the GDA model was easily generated using ATP-Draw, a graphical mouse-driven preprocessor for ATP; it estimates the breakdown characteristics of GDAs with source triggers. The ATP-EMTP model of the GDA (Fig. 9) is composed of four parts. The surge voltage generator produced the standard $1.2 / 50 \mu \mathrm{s}$ lightning impulse voltage for the open circuit and a standard $8 / 20 \mu$ s current for the short circuit. Measurements were collected with a shunt resistor and a shunt capacitor.

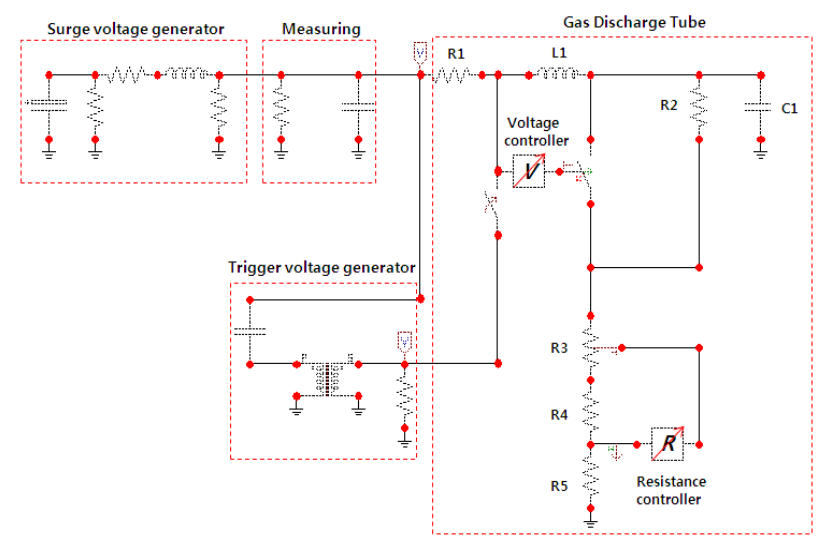

Fig. 9. ATP-Draw picture of the GDA model. 
These elements for measurement did not influence the obtained results because resistance has a much higher value than the parallel resistor of the generator. Moreover, the capacitance was too small to affect significantly the steepness of the voltage [5], [8]. The trigger voltage generator was applied to the middle electrode of the GDA. The right portion of the model corresponds to the GDA itself, while time delay is represented by a voltage controller. Eq. (1) was implemented using the MODELS function. The plasma resistance is represented by a resistance controller. The resistance controller box utilized a MODELS module implementing Eq. (2) and which considers voltage as input (the current can be obtained by dividing voltage by measured resistance $\mathrm{R}_{5}$ ). In the model, $\mathrm{R}_{2}$ and $\mathrm{C}_{1}$ are the leakage resistance and the capacitance of the GDA, respectively. $\mathrm{L}_{1}$ is the inductance of the leads. $R_{4}$ is the contact resistance and $R_{1}$ is a negligible resistor that has been introduced in order to create a node inside the GDA module connected to the voltage controller.

Fig. 10 shows the simulation results for an applied impulse voltage wave with a slope of $18.5 \mathrm{kV} / \mu$ s and peak voltage of $4 \mathrm{kV}$. Upon application of the impulse voltage to the GDA, the secondary voltage of the transformer decreased as oscillation decayed. These results were compared with those in Fig. 4 where the slope of the voltage wave has been amplified by the capacitor and transformer, and at which voltages were measured with additional $200 \mathrm{~ns}$. The obtained results were similar to those shown in Fig. 4.

Fig. 11 shows the GDA operation characteristics when the trigger voltage is applied and not applied with a slope of $18.5 \mathrm{kV} / \mu \mathrm{s}$ and a peak voltage of $4 \mathrm{kV}$.

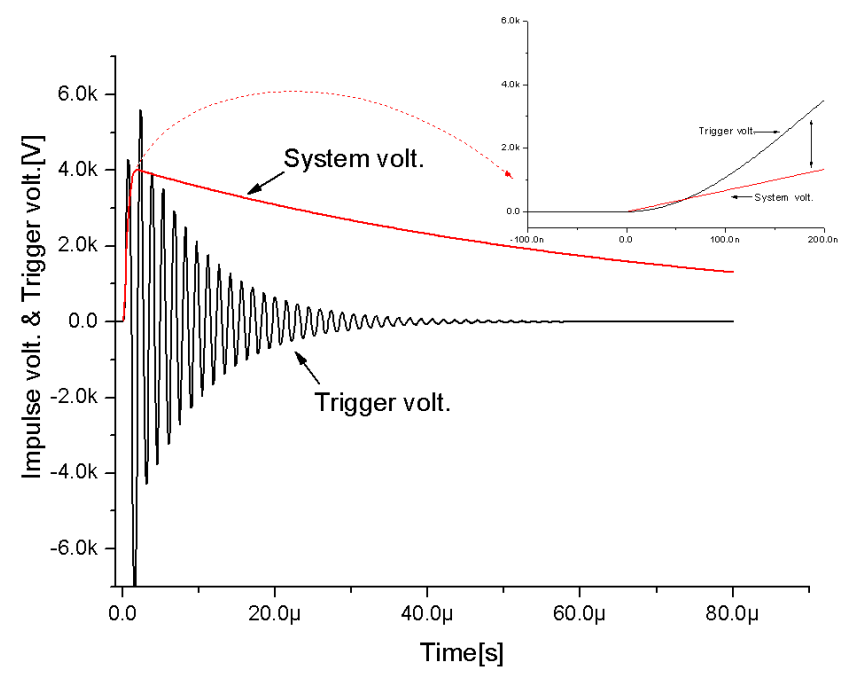

Fig. 10. Simulated test voltage applied to the GDA.

Fig. 11(a) shows as case where the trigger voltage is not applied to the middle electrode of the GDA, whereas in Fig. 11(b), trigger voltage is applied. A comparison of simulation results and the experimental results is shown in Table 2. Evidently, as the difference between the results is less than $2 \%$ upon inducing the trigger source, this model can be used to estimate the breakdown characteristics of GDAs.
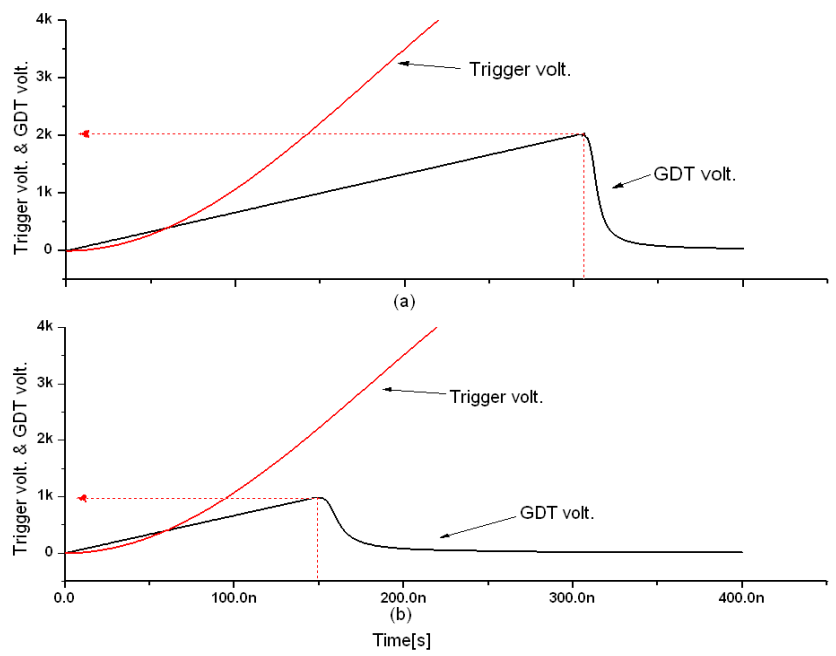

Fig. 11. GDA operation with trigger voltage at $18.5 \mathrm{kV} / \mu \mathrm{s}$ and $4 \mathrm{kV}_{\max }$.

Table 2. Comparison of simulation and experimental results

\begin{tabular}{c|c|c|c|c}
\hline Condition & Factors & $\begin{array}{c}\text { Experiment } \\
\text { (Figs. 8 and 9) }\end{array}$ & $\begin{array}{c}\text { Simulation } \\
\text { (Fig. 12) }\end{array}$ & $\Delta \%$ \\
\hline \multirow{2}{*}{$\begin{array}{c}\text { Without } \\
\text { trigger }\end{array}$} & Voltage (V) & 2356 & 2043 & 13.3 \\
\cline { 2 - 5 } & Time (ns) & 225 & 306 & 36 \\
\hline \multirow{2}{*}{$\begin{array}{c}\text { With } \\
\text { trigger }\end{array}$} & Voltage (V) & 998 & 984 & 1.4 \\
\cline { 2 - 5 } & Time (ns) & 146 & 149 & 2.0 \\
\hline
\end{tabular}

\section{Conclusion}

1. Response time decreases as the slope of the applied impulse voltage increases. However, the commensurate increase in breakdown voltage caused by this time increase can damage devices that are sensitive to voltage surges. In this paper, the high voltage amplified and synchronized from the test voltage is applied to the middle electrode as a trigger. As a result, the time to discharge and the starting breakdown voltage decreased.

2. In applying the trigger source, the slope of the trigger voltage is increased to 6.2 times than the slope of the test voltage. Therefore, using a trigger electrode enables breakdown voltage to decrease from $45 \%$ to $57.6 \%$

3. If faster sensing of the applied surge and a higherinduced $\mathrm{dv} / \mathrm{dt}$ of the applied surge are triggered at the middle electrode, a lower breakdown voltage is achieved.

4. A GDA model based on the experimental results has been developed. This model, created using ATP-Draw, is more convenient to use than the conventional method and can be used to calculate GDA operation characteristics. In this paper, when the trigger source is used, the actual test results and the simulation results agrees within a $2 \%$ range. 


\section{References}

[1] A.Torrelli, S. Pappas, "An update on power line surge protection techniques for telecommunication facilities," Telecommunications Energy Conf., 2008.

[2] R. B. Standler, "Protection of Electronic Circuits from over voltages," Dover Edition, 2002.

[3] R. Rosen, "Application of carbon nanotubes as electrodes in gas discharge tubes," Appl.Phys. Lett., Vol 76, 2000, pp. 1668-1670.

[4] A. Larsson, V. Scuka, K. Borgeest, J. L. Haseborg, "Numerical Simulation of Gas Discharge Protectors A Review," IEEE Trans. Power Del., Vol. 14, 1999, pp. 405-410.

[5] A. Larsson, H. Tang, and V. Scuka, "A Gas Discharge Tube Module based on Discharge Physics for use in ATP-EMTP Simulations," EEUG News Nov., 1995, pp. 55-61.

[6] "Surge arresters and switching spark gaps," www.epcos.com, 2009.

[7] H. Golnabi, "Discharge Current Development in Two-Electrode Spark Gap Switches," IEEE Trans. Plasma Sci., Vol. 30, 2002, pp. 301-309.

[8] A. Larsson, H. Tang, and V. Scuka, "Mathematical simulation of a gas discharge protector using ATPEMTP," in Proc. Int. Symp. EMC, Rome, Italy, 1996, pp. 315-320.
[9] S. Singha and M. J. Thomas, "Toepler's Spark Law in a GIS with compressed $\mathrm{SF}_{6}-\mathrm{N}_{2}$ mixture," IEEE Trans. Dielectr. Electr. Insul., Vol. 10, 2003, pp. 498-505.

[10] P. H. Schavenmaker and L. van der Sluis, "An improved Mayr-Type Arc model based on current zero measurements," IEEE Trans. Power Del., Vol. 15, 2000, pp. 580-584.

[11] P. Seers, "Spark ignition: An experimental and numerical investigation," Ph.D. dissertation, Univ. Texas, Austin, 2003.

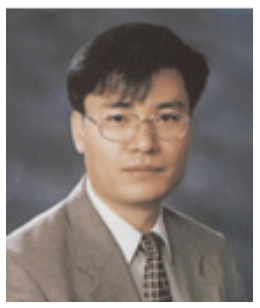

Sei-Hyun Lee was born in Daejeon, Korea in 1967. He received the B.Sc., M.Sc., and $\mathrm{PhD}$ in electrical engineering from the Chungnam National University, Korea in 1992, 1994, and 1998, respectively. He joined the Korea Polytechnic College 4 where he has been an associate professor of Electrical Measurement and Control Engineering since 1997. He was with the University of New South Wales (UNSW), as a postdoctorial fellow from 2003 to 2004. His main research interests are surge suppressors, lightning arresters, high voltage power fuses, and switchgears. 